\title{
Ge Outdiffusion Effect on Flicker Noise in Strained-Si nMOSFETs
}

\author{
W.-C. Hua, M. H. Lee, P. S. Chen, S. Maikap, C. W. Liu, Senior Member, IEEE, and K. M. Chen
}

\begin{abstract}
The flicker noise characteristics of strained-Si nMOSFETs are significantly dependent on the gate oxide formation. At high temperature $\left(900{ }^{\circ} \mathrm{C}\right)$ thermal oxidation, the Si interstitials at the $\mathrm{Si} /$ oxide interface were injected into the underneath $\mathrm{Si}-\mathrm{SiGe}$ heterojunction, and enhanced the Ge outdiffusion into the $\mathrm{Si} /$ oxide interface. The $\mathrm{Ge}$ atoms at $\mathrm{Si} /$ oxide interface act as trap centers, and the strained-Si nMOSFET with thermal gate oxide yields a much larger flicker noise than the control $\mathrm{Si}$ device. The Ge outdiffusion is suppressed for the device with the low temperature $\left(700{ }^{\circ} \mathrm{C}\right)$ tetraethylorthosilicate gate oxide. The capacitance-voltage measurements of the strained-Si devices with thermal oxide also show that the $\mathrm{Si} /$ oxide interface trap density increases and the $\mathrm{Si}$-SiGe heterojunction is smeared out due to the Ge outdiffusion.
\end{abstract}

Index Terms-Flicker noise, Ge outdiffusion, strained-Si, MOSFET.

\section{INTRODUCTION}

D UE TO the mobility enhancement of strained-Si channels, the strained-Si MOSFETs have made great improvements on dc characteristics [1], [2]. The conventional method to obtain the strain in $\mathrm{Si}$ is to utilize the lattice misfit between the relaxed $\mathrm{SiGe}$ and $\mathrm{Si}$, and the $\mathrm{Si}-\mathrm{SiGe}$ heterojunction is formed unintentionally underneath the device channel. The role of Ge may lead to the different electrical characteristics of the strained-Si devices. For the radio frequency applications, the low frequency noise behavior of the active device has great influence on the phase noise of an oscillator, but few literatures are on this issue. In this letter, the flicker $(1 / f)$ noise of the strained-Si nMOSFETs is investigated. As a result, the device process has significant effect on the flicker noise.

\section{DEVICE FABRICATION}

The schematic structure of the strained-Si nMOSFET is illustrated in Fig. 1. The $\mathrm{Si}_{1-x} \mathrm{Ge}_{x}$ graded buffer layer was expitaxially grown on a p-type Si substrate at $600{ }^{\circ} \mathrm{C}$. Silane and germane were used as the $\mathrm{Si}$ and Ge precursors, respectively, and the Ge content of the graded buffer increased

Manuscript received May 7, 2004. This work was supported by the National Science Council of Taiwan, R.O.C. under Contract 92-2215-E-002-007 and Contract 92-2120-E-002-006. The review of this letter was arranged by Editor T.-J.King.

W.-C. Hua, S. Maikap, and C. W. Liu are with the Department of Electrical Engineering, Graduate Institute of Electronics Engineering, National Taiwan University, Taipei, Taiwan, R.O.C. (e-mail: chee@cc.ee.ntu.edu.tw).

M. H. Lee and P. S. Chen are with the Electronics Research and Service Organization, Industrial Technology Research Institute (ERSO/ITRI), Hsinchu 300, Taiwan, R.O.C.

K. M. Chen is with the National Nano Device Laboratories, Hsinchu 300, Taiwan, R.O.C.

Digital Object Identifier 10.1109/LED.2004.834884

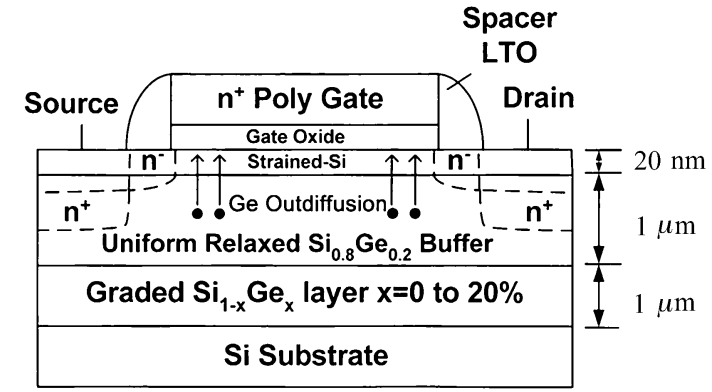

Fig. 1. Schematic diagram of a strained-Si nMOSFET.

from $0 \%$ to $20 \%$ over the thickness of $1 \mu \mathrm{m}$. A $1-\mu \mathrm{m}$-thick relaxed $\mathrm{Si}_{0.8} \mathrm{Ge}_{0.2}$ layer was grown on the graded buffer layer, and a $20-\mathrm{nm}$ strained-Si layer was grown on the uniform relaxed $\mathrm{Si}_{0.8} \mathrm{Ge}_{0.2}$ layer. The surface roughness of the resulting strained-Si is $\sim 5 \mathrm{~nm}$ after the device fabrication, measured by the atomic force microscope. All the epitaxial layers were prepared by the ultrahigh-vacuum chemical vapor deposition reactor. Both the strained-Si and the control Si nMOSFETs were fabricated by the same process with the $25-\mu \mathrm{m}$ gate width and the $0.8-\mu \mathrm{m}$ gate length. The maximum thermal budget is $960^{\circ} \mathrm{C}$ for $7 \mathrm{~min}$. The tensile strain in the $\mathrm{Si}$ channel is $\sim 0.64 \%$, measured by surface Raman spectroscopy [3]. The treading dislocation density on the strained-Si is $\sim 10^{6} \mathrm{~cm}^{-2}$, and almost no dislocation penetrates into the device with $L=0.8 \mu \mathrm{m}$ and $W=25 \mu \mathrm{m}$. The effect of the threading dislocation can be neglected in these devices.

\section{RESULTS AND DISCUSSION}

The normalized input referred gate voltage noises $\left(\mathrm{NS}_{\mathrm{Vg}}\right)$ for both the strained-Si and the control Si nMOSFETs with rapid thermal oxidation (RTO) $\left(5 \mathrm{~nm}\right.$ at $900{ }^{\circ} \mathrm{C}$ for $\left.5 \mathrm{~min}\right)$ and deposited tetraethylorthosilicate (TEOS) oxide $\left(30 \mathrm{~nm}\right.$ at $700{ }^{\circ} \mathrm{C}$ for $1 \mathrm{~h}$ ) are shown in Fig. 2. $\mathrm{NS}_{\mathrm{Vg}}$ is independent of oxide thickness and is given by

$$
\mathrm{NS}_{\mathrm{Vg}}=\frac{S_{\mathrm{Vg}}}{t_{\mathrm{ox}}^{2}}=\frac{S_{I d}}{g_{m}^{2} t_{\mathrm{ox}}^{2}}=\frac{q^{2}}{\varepsilon_{\mathrm{ox}}^{2}} \frac{N_{\mathrm{ot}}}{W L} \frac{1}{f}
$$

where $S_{\mathrm{Vg}}$ is the input referred gate voltage noise, $S_{I d}$ is the drain current noise, $g_{m}$ is the transconductance, $\varepsilon_{\mathrm{ox}}$, and $t_{\mathrm{ox}}$ are the dielectric constant and thickness of the gate oxide, respectively. $N_{\text {ot }}$ is the equivalent oxide traps per unit area, $W$ and $L$ are channel width and length, respectively, and $f$ is frequency. Equation (1) is based on the carrier number fluctuation model [4]-[6] also known as the trapping-detrapping model originated by McWhoter [4], [5] for the nMOSFET. The effect of the oxide thickness on the flicker noise can be normalized in (1). All the 


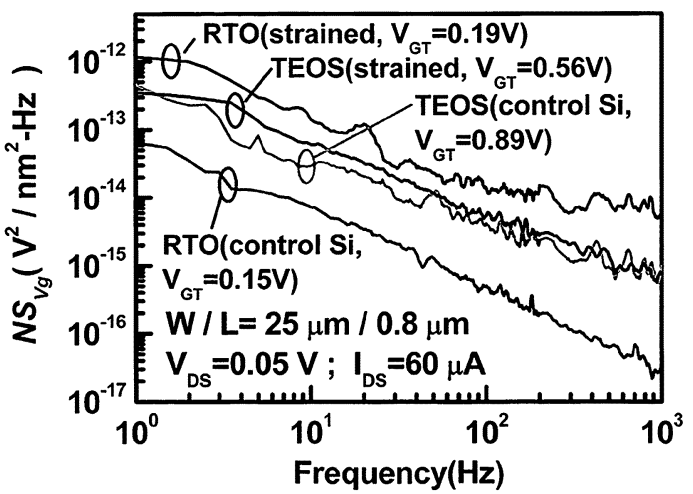

Fig. 2. $\mathrm{NS}_{\mathrm{Vg}}$ of the strained-Si and the control Si devices with RTO and TEOS oxide. The strained-Si device with RTO gate oxide has a much higher $\mathrm{NS}_{\mathrm{Vg}}$ than the control device with RTO, but the strained-Si device with TEOS gate oxide shows slightly increased $\mathrm{NS}_{\mathrm{Vg}}$ than the control device.

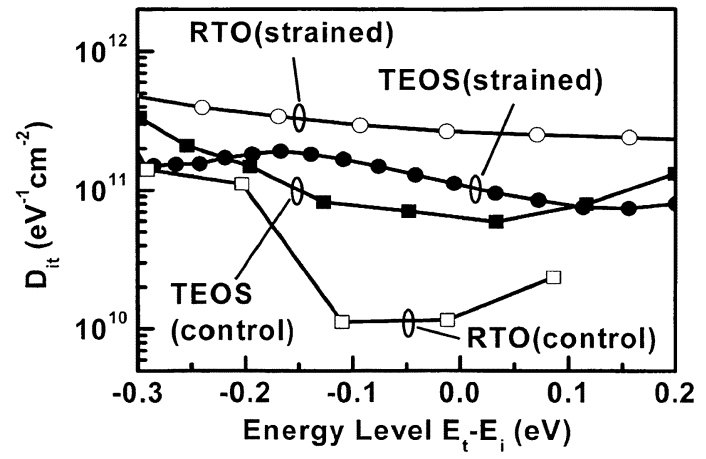

Fig. 3. $D_{\text {it }}$ of the strained-Si and control Si devices with TEOS and RTO oxide. The strained-Si device with RTO oxide has the highest $D_{\text {it }}$ of $5 \times 10^{11}$ $\mathrm{eV}^{-1} \mathrm{~cm}^{-2}$ due to the oxidation-enhanced Ge outdiffusion.

devices are biased at triode region with the same $I_{\mathrm{DS}}(60 \mu \mathrm{A})$ and very small $V_{\mathrm{DS}}(0.05 \mathrm{~V})$ to maintain the similar condition as the $D_{\text {it }}$ extraction measurement (no lateral electric field in the channel). Even though the four devices are biased at different effective gate voltages $\left(V_{\mathrm{GT}}\right), \mathrm{NS}_{\mathrm{Vg}}$ is still comparable since the $S_{I d}$ is normalized to the square of the transconductance $\left(V_{\mathrm{GT}}\right.$ dependence). Note that $N_{\text {ot }}$ is only weakly dependent on the gate voltage when $V_{\mathrm{GT}}>0$ [6]. The measured transconductance is used in the $\mathrm{NS}_{\mathrm{Vg}}$ calculation. Fig. 2 shows that for RTO gate oxide, the strained-Si MOSFET yields a higher $\mathrm{NS}_{\mathrm{Vg}}$ than the control device at the same frequency, but for the TEOS gate oxide, the strained-Si MOSFET exhibits only a slightly higher $\mathrm{NS}_{\mathrm{Vg}}$ than the control one. The results can be explained by the carrier number fluctuation theory and the Ge outdiffusion [7]. Since the $\mathrm{NS}_{\mathrm{Vg}}$ is proportional to the $N_{\mathrm{ot}}$ in (1) and the $N_{\text {ot }}$ is approximately proportional to the interface trap densities $\left(D_{\mathrm{it}}\right)$, the $\mathrm{NS}_{\mathrm{Vg}}$ can be directly related to the $D_{\mathrm{it}}$. The $D_{\mathrm{it}}$ of the four devices can be obtained from the high-low frequency capacitance-voltage ( $C-V$ ) (Fig. 3) [8]. The order of the $\mathrm{NS}_{\mathrm{Vg}}$ level in Fig. 2 is consistent with the $D_{\text {it }}$ level in Fig. 3. For the control Si devices, the RTO device has lower $D_{\text {it }}$ and $\mathrm{NS}_{\mathrm{Vg}}$ as compared to the TEOS device due to the high quality thermal oxide formation at $900^{\circ} \mathrm{C}$. However, the strained-Si device with RTO oxide has the highest $D_{\text {it }}$ and $\mathrm{NS}_{\mathrm{Vg}}$ due to the oxidation-enhanced Ge outdiffusion. At the high temperature RTO process, the $\mathrm{Si}$-interstitials at $\mathrm{Si} /$ oxide interface were injected

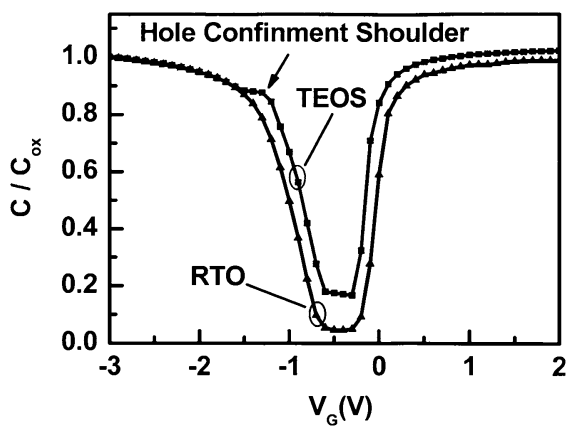

Fig. 4. Room temperature quasi-static $C-V$ curves of strained-Si devices with TEOS and RTO oxide.

into the underneath $\mathrm{Si}-\mathrm{SiGe}$ heterojunction, and the interstitials enhanced the $\mathrm{Ge}$ outdiffusion in the relaxed $\mathrm{SiGe}$ layer into the strained-Si/oxide interface. The Ge acts as a trap center. The traps near the strained-Si/oxide interface enhance the random trapping and detrapping processes of charges. The charge fluctuation induces the fluctuation of the surface potential and modulates the channel carrier density. As a result, the flicker noise of the strained-Si device with RTO oxide increases. The saturation drain current $I_{\text {Dsat }}$ of the strained-Si device has a $35 \%$ increase and a $-42 \%$ decrease at $V_{\mathrm{GS}}-V_{\mathrm{t}}=3 \mathrm{~V}$ as compared to the control device for TEOS and RTO oxides, respectively. The decrease of $I_{\text {Dsat }}$ of the strained-Si device with RTO oxide as compared to the control device with the same RTO oxide also suggests that Ge traps can decrease the electron mobility. The mobility enhancement obtained by low temperature process has been reported previously [9]. Both the $\mathrm{NS}_{\mathrm{Vg}}$ and $D_{\text {it }}$ are similar for the strained-Si and control Si devices with low temperature TEOS oxide. This indicates that for the low temperature (TEOS) process, the Ge outdiffusion at $700{ }^{\circ} \mathrm{C}$ is not significant and the interface trap densities are almost similar for both the strained-Si and the control Si devices. Note that the Ge diffusion at $950{ }^{\circ} \mathrm{C}$ without $\mathrm{Si}$-interstitial injection can be enhanced for strained-Si [10]. This may be responsible for the slightly higher $\mathrm{NS}_{\mathrm{Vg}}$ and $D_{\text {it }}$ of the strained-Si device with TEOS oxide as compared to the control Si device with the same TEOS oxide.

To further confirm the Ge outdiffusion, quasi-static $(C-V)$ measurements of strained-Si devices with the TEOS and RTO oxide at room temperature are performed (Fig. 4). For the strained-Si device with TEOS gate oxide, the hole confinement shoulder due to the strained-Si/relaxed $\mathrm{SiGe}$ heterojunction [11] is clearly observed in the $C-V$ curves at the accumulation region $\left(V_{\mathrm{G}}=-1 \mathrm{~V}\right)$. The existence of the hole confinement shoulder is confirmed by quasi-static $C-V$ measurements from room temperature to $230{ }^{\circ} \mathrm{C}$. However, for the strained-Si device with $\mathrm{RTO}$ gate oxide, no such hole confinement shoulder is observed. This indicates that the strained-Si/relaxed $\mathrm{SiGe}$ heterojunction of the RTO device is smeared out due to the Ge outdiffusion during the RTO process.

\section{CONCLUSION}

Both the disappearance of hole confinement shoulder in the $C-V$ measurements and the increase of $D_{\text {it }}$ and $\mathrm{NS}_{\mathrm{Vg}}$ for the strained-Si device with RTO oxide indicate the Ge outdiffusion from the $\mathrm{Si}-\mathrm{SiGe}$ heterojunction which smears out the 
abruptness of the heterojunction, and forms interface traps at the $\mathrm{Si} /$ oxide interface. The low temperature oxide is required to avoid the injection of Si-interstitials and the Ge outdiffusion.

\section{REFERENCES}

[1] J. L. Hoyt, H. M. Nayfeh, S. Eguchi, I. Aberg, G. Xia, T. Drake, E. A. Fitzgerald, and D. A. Antoniadis, "Strained silicon MOSFET technology," in IEDM Tech. Dig., 2002, pp. 23-26.

[2] K. Rim, S. Narasimha, M. Longstreet, A. Mocuta, and J. Cai, "Low field mobility characteristics of sub-100 nm unstrained and strained Si MOSFETs," in IEDM Tech. Dig., 2002, pp. 43-46.

[3] J. C. Tsang, P. M. Mooney, F. Dacol, and J. O. Chu, "Measurements of alloy composition and strain in thin $\mathrm{Ge}_{x} \mathrm{Si}_{1-x}$ layers," J. Appl. Phys., vol. 75, no. 12, pp. 8098-8108, 1994.

[4] A. L. McWhorter, "Semiconductor surface physics," Ph.D. dissertation, Lincoln Lab., Mass. Inst. Technol., Lexington, MA, 1955.
[5] A. L. McWhorter, Semiconductor Surface Physics, R. H. Kinston, Ed. Philadelphia, PA: Univ. Penn., 1957.

[6] Y. Nemirovsky, I. Brouk, and C. G. Jakobson, " $1 / f$ noise in CMOS transistors for analog applications," IEEE Trans. Electron. Devices, vol. 48, pp. 921-927, May 2001.

[7] P. Fahey, S. S. Lyer, and G. J. Scilla, "Experimental evidence of both interstitial- and vacancy-assisted diffusion of Ge in Si," Appl. Phys. Lett., vol. 54, no. 9, pp. 843-845, 1989.

[8] E. H. Nicollian and J. R. Brews, MOS (Metal Oxide Semiconductor) Physics and Technology. New York: Wiley, 1991.

[9] K. Rim, J. L. Hoyt, and F. Gibbons, "Fabrication and analysis of deep submicron strained-Si N-MOSFETs," IEEE Trans. Electron Devices, vol. 47, pp. 1406-1415, July 2000.

[10] T. Mizuno, N. Sugiyama, T. Tezuka, T. Numata, T. Maeda, and S. Takagi, "Design for scaled thin film strained-SOI CMOS devices with higher carrier mobility," in IEDM Tech. Dig., 2002, pp. 31-34.

[11] G. A. Armstrong and C. K. Majti, "Strained-Si channel heterojunction PMOSFETs," Solid State Electron., vol. 42, no. 4, pp. 487-498, 1998. 